Mônica Angelim Gomes de Lima ${ }^{1}$ Andréa Garboggini Melo Andrade2.3 Camila Moitinho de Aragão Bulcão ${ }^{2}$ Esperança Maria de Carvalho Lino Mota ${ }^{2}$ Francesca de Brito Magalhães 2,3 Rita de Cássia Peralta Carvalho ${ }^{2}$ Sonara Machado de Carvalho Freitas ${ }^{2}$ Sylvia Regina Freire de Carvalho Sá ${ }^{2}$ Lauro Antonio Porto ${ }^{1}$

Robson da Fonseca Neves ${ }^{4}$

${ }^{1}$ Departamento de Medicina Preventiva e Social, Faculdade de Medicina da Bahia, Universidade Federal da Bahia.

${ }^{2}$ Centro Estadual de Referência em Saúde do Trabalhador da Bahia (Cesat/ Bahia).

${ }^{3}$ Escola Bahiana de Medicina e Saúde Pública, Curso de Terapia Ocupacional. ${ }^{4}$ Departamento de Fisioterapia da Universidade Federal da Paraíba.

Contato:

Mônica Angelim Gomes de Lima

Endereço: Universidade Federal da Bahia, Faculdade de Medicina da Bahia, Departamento de Medicina Preventiva e Social.

Avenida Reitor Miguel Calmon s/n

- Canela - Salvador - BA.

CEP: 40110-100

E-mail:

angelim@ufba.br

Recebido: 30/06/2009

Revisado: 05/02/2010

Aprovado: 09/02/2010

\section{Programa de reabilitação de trabalhadores com LER/ DORT do Cesat/Bahia: ativador de mudanças na Saúde do Trabalhador}

\author{
Cesat/Bahia rehabilitation program for workers with RSI/ \\ WRMD - a starter for changes in Workers' Health
}

\section{Resumo}

Este artigo apresenta a concepção e a implantação do Programa de Retorno ao Trabalho (PRT), em fase de projeto piloto, como parte do Programa de Reabilitação de Trabalhadores com LER/DORT do Cesat/Bahia, desde 2007. Esta experiência tem-se apresentado como um processo ativador de diálogos e mudanças no campo da saúde do trabalhador no estado da Bahia, envolvendo os diversos atores implicados na reabilitação de trabalhadores. É apresentado o GT de Reabilitação/Cesat, sua constituição, composição e características de atuação, os caminhos trilhados, as escolhas feitas no sentido da integração das ações de vigilância e assistência a partir do PRT/Cesat/BA. São descritas suas etapas, o papel de cada ator envolvido, os métodos e instrumentos utilizados e os resultados esperados. Chama-se atenção para a aproximação com a CIF enquanto um recurso para dar visibilidade às demandas biopsicossociais do processo de saúde e doença dos trabalhadores. A elaboração do PRT tem proporcionado o amadurecimento teórico do grupo técnico, como também o fortalecimento das interações institucionais necessárias ao desenvolvimento de um programa de reabilitação de trabalhadores de abrangência estadual, contribuindo para a formatação de ações mais integradoras para o enfrentamento da complexa problemática da reabilitação de trabalhadores na Renast/SUS.

Palavras-chave: reabilitação de trabalhadores; programa de retorno ao trabalho; funcionalidade; CIF; LER/DORT.

\begin{abstract}
This article presents the formulation and adoption of the "Programa de Retorno ao Trabalho" (Return to Work Program - PRT), still in a pilot project stage, as part of Cesat/Bahia (Occupational Health Reference Center of the state of Bahia, Brazil) Rehabilitation Program for workers with RSI/WMSD. This experience has fostered debates and changes within the occupational health field in the state of Bahia, involving several agents engaged with workers' rehabilitation. The paper introduces the Cesat Rehabilitation Work Group, its constitution, composition, and operational characteristics, as well as paths followed and choices made aiming the integration of surveillance and healthcare actions in PRT since 2007. It describes all the stages of the program, the role of each agent involved, methods and instruments used, and the expected results. It emphasizes the use of ICF (International Classification of Functioning, Disability and Health) as a resource to give visibility to the biopsychosocial demands of workers' health/disease process. The formulation of PRT has promoted the theoretical development of the technical group and strengthened the institutional interactions needed to establish a statewide Workers' Rehabilitation Program. It has also contributed to the development of more integrative actions in order to face the complex problems concerning workers' rehabilitation in the National Network for Comprehensive Workers Healthcare (Renast) within the National Health System (SUS).
\end{abstract}

Keywords: workers' rehabilitation; return to work program; functioning; ICF; $R S I / W M S D$. 


\section{Introdução}

A reabilitação de trabalhadores é um dos maiores desafios contemporâneos para o campo da saúde pública no Brasil. A complexidade e as tensões que emergem nesta problemática refletem a multiplicidade e a magnitude dos problemas de saúde e as repercussões sociais envolvidas, ao tempo em que impõem abertura de agenda pública específica, construção de novas práticas e formulação de políticas públicas abrangentes que compreendam e enfrentem a complexidade do problema. (MAENO, 2001; MAENO; TAKAHASHI; LIMA, 2009).

O crescimento da notificação e dos afastamentos do trabalho decorrentes de doenças ocupacionais, a partir da década de 1990, aumentaram também a complexidade da discussão sobre a capacidade/incapacidade para o trabalho, tanto na dimensão técnica, requerendo novas soluções tecnológicas, como na dimensão sociopolítica, implicando novos arranjos institucionais e relações interinstitucionais, especialmente considerando as mudanças ocorridas no interior da Previdência Social neste período, assim como as ações insuficientes sobre este tema no campo da Saúde do Trabalhador (ST), no Sistema Único de Saúde (SUS) e no movimento social (TAKAHASHI; IGUTI, 2008; SOUZA; ESTRELA, 2003).

Precisam ser consideradas também as especificidades do contexto brasileiro para o enfrentamento do processo diagnóstico-tratamento-reabilitação de trabalhadores, que incluem: as características dos trabalhadores com LER/DORT que buscam a Renast/SUS; as dificuldades enfrentadas para a implantação de ações de vigilância de processos de trabalho com modificação efetiva de condições e organização do trabalho e o acompanhamento deste processo dentro das empresas; e o distanciamento político-institucional do principal sistema de compensação: a Previdência Social.

Um momento recente importante para a discussão da reabilitação de trabalhadores no Brasil foi o Seminário "Reabilitação profissional pública: um direito do cidadão”, realizado na Fundacentro-SP em outubro/2007 (ROSSI et al., 2007). Este evento reuniu técnicos da área de saúde do trabalhador e da Previdência Social com a finalidade de refletir e propor um novo modelo de reabilitação a ser adotado pela Previdência Social. Defendeu, centralmente, a construção de ações integradas ao SUS, permitindo um fluxo complementar entre a Previdência e os demais setores do Estado envolvidos com a atenção da saúde dos trabalhadores.

Assume-se neste artigo a perspectiva da vigilância à saúde como proposta de ação, entendendo que, para responder às necessidades sociais que emergem do processo de reabilitação de trabalhadores, é necessária a articulação de ações individuais e coletivas, institucionais e interinstitucionais, setoriais e transetoriais (PAIM, 2002; MAENO; TAKAHASHI; LIMA, 2009) e a busca da integralidade da atenção, enquanto caminho a ser trilhado, reflexo dos consensos construídos ao longo do tempo (XAVIER; GUIMARÃES; PINHEIRO, 2004).
Neste artigo, apresenta-se o processo de concepção e implantação do Programa de Retorno ao Trabalho (PRT), em fase de projeto piloto, como parte do Programa de Reabilitação de Trabalhadores com LER/DORT do Centro Estadual de Referência em Saúde do Trabalhador - Cesat/Bahia.

\section{Método}

Trata-se de um relato de experiência do processo de constituição do Programa de Retorno ao Trabalho (PRT) do Cesat/BA, apresentando desde a formação de Grupo de Trabalho (GT Reabilitação), a partir de 2007, até o PRT proposto: suas etapas, o papel de cada ator envolvido, os métodos e instrumentos utilizados e os resultados esperados com o objetivo de permitir a reprodução desta experiência em outros Centros de Referência em Saúde do Trabalhador (Cerest) e as aproximações feitas com a Classificação Internacional de Funcionalidade, Incapacidade e Saúde - CIF (ORGANIZAÇÃO MUNDIAL DA SAÚDE, 2003) e com a literatura internacional.

\section{GT Reabilitação do Cesat - formação e composição}

O progressivo aumento do diagnóstico das LER/ DORT associadas às Doenças da Coluna Lombar registrado no período de 1991 a 2000 (SOUZA; ESTRELA, 2003) e os consequentes afastamentos temporário e permanente do trabalho contribuíram para que, em 1998 (BAHIA, 2002), fossem iniciadas, no Cesat, ações voltadas para o cuidado de trabalhadores. Através da iniciativa de terapeutas ocupacionais, fisioterapeutas, médico, psicoterapeuta e assistentes sociais, foram estruturadas atividades de educação para saúde e atendimentos grupais - Grupo de Qualidade de Vida, Grupo de Terapia Ocupacional, Oficina de Autocuidado e Grupo Respirar.

Os pressupostos assumidos nesses trabalhos eram direcionados para o resgate da saúde, aqui entendida no seu sentido mais amplo e não apenas voltada para o retorno do trabalhador à produção. Tinham-se como foco as questões coletivas e as suas repercussões em cada indivíduo, visando à construção de um projeto terapêutico integrado que contribuísse para a ressignificação da doença e a ampliação da autonomia do trabalhador nas suas atividades cotidianas.

Esses grupos constituíram-se como espaços de troca de experiências, de aprendizado e de possibilidades de mudanças. Estimulavam-se a comunicação entre os participantes e a aquisição de conhecimento a respeito do trabalho, seus riscos e mecanismos adoecedores; possibilitavam-se o autoconhecimento e o estabelecimento de limites, propiciando a ampliação da capacidade funcional, incluindo atividades ocupacionais; buscava-se o redimensionamento dos sintomas físicos e psíquicos e o ajuste do uso de medicações analgésicas e de psicofármacos; e construíam-se, coletivamente, estratégias para realização das atividades da vida diária com menor dispêndio de energia. 
Apesar de não serem desenvolvidas ações direcionadas para o retorno ao trabalho, a aflição dos trabalhadores frente à iminência da alta do INSS era tema frequentemente discutido nos diversos grupos. A ansiedade gerada pode ser entendida, conforme Takahashi e Iguti (2008), pelo fato de não lhes ter sido ofertada, através de programas de reabilitação profissional, a possibilidade de superação de suas desvantagens.

Nesse contexto, alguns integrantes dos atendimentos grupais, que também faziam parte de um grupo de discussão sobre LER do Sindicato dos Bancários da Bahia, decidiram, em 1995, formar uma comissão de pessoas com LER/DORT, envolvendo diversas categorias (bancários, metalúrgicos, químicos, telefônicos, comerciários, trabalhadores em processamento de dados). Tal comissão, posteriormente, passou a ser denominada Comissão Intersindical de LER. Já em 1996, a Comissão lançou, na Câmara de Vereadores de Salvador, uma campanha de combate e prevenção a LER, buscando dar visibilidade a este agravo. Em 1999, diante da necessidade de legalizar o movimento, foram iniciadas as discussões para elaboração do estatuto do Centro de Estudos, Prevenção e Apoio aos Portadores de LER/DORT (CEAPLER), culminando com a sua fundação em 12 de novembro de 2001.

A avaliação das atividades desenvolvidas nos atendimentos grupais, bem como o cotidiano de trabalho dos profissionais do centro, fomentaram discussões na equipe técnica sobre a necessidade do desenvolvimento de um programa de reabilitação para trabalhadores com LER/DORT que contemplasse a complexidade deste agravo em todas as suas dimensões: física, psíquica, social e histórica.

Como resposta às inquietações geradas por esta realidade, constituiu-se, em setembro de 2007, um grupo de trabalho - GT Reabilitação do Cesat, com o objetivo de desenvolver uma proposta de Reabilitação de Trabalhadores com LER/DORT possível de ser implementada na Rede Nacional de Atenção Integral à Saúde do Trabalhador - Renast/SUS. Esse grupo é composto por técnicos que atuam nas coordenações de Atenção Integral à Saúde do Trabalhador (COAST) e de Vigilância de Ambientes e Processos de Trabalho (COVAP), das áreas de Fisioterapia, Medicina, Serviço Social e Terapia Ocupacional, com especialização em Saúde do Trabalhador, Ergonomia, Dor Crônica e Psicoterapia.

O GT Reabilitação tem conhecido a literatura que discute modelos de retorno ao trabalho, investindo em processo contínuo de reflexão e educação permanente com a contribuição da universidade (LOISEL et al., 1994; DURAND et al., 2003). É consenso que a pre- venção da incapacidade e a reabilitação de trabalhadores são diretamente proporcionais à capacidade de intervenção no indivíduo e no coletivo, promovendo mudanças nos processos de trabalho, na sua dimensão material e no contexto sociocultural com base em abordagens compreensivas e interdisciplinares (SCHULTZ et al., 2005; YOUNG et al., 2005).

O primeiro parceiro do GT Reabilitação tem sido o Grupo de Pesquisa do CNPq Saúde, Trabalho e Funcionalidade $^{5}$, coordenado pelo Departamento de Medicina Preventiva e Social da Faculdade de Medicina da Bahia/UFBA. Esta parceria vem sendo progressivamente ampliada com as aproximações sucessivas da problemática que envolve a reabilitação de trabalhadores, iniciando-se com a apresentação à Classificação Internacional de Funcionalidade, Incapacidade e Saúde - CIF (ORGANIZAÇÃO MUNDIAL DA SAÚDE, 2003) - e ao Protocolo de Avaliação da Funcionalidade de Trabalhadores com LER/DORT proposto pelo grupo de pesquisa para o GT Reabilitação.

A problematização dos caminhos escolhidos reiterou a necessidade de efetivar um estudo de caso que permitisse pôr em prática a experiência compartilhada por outros centros de referência (Cerest Campinas e Piracicaba) e vários autores (TAKAHASHI; CANESQUI, 2003; TAKAHASHI; IGUTI, 2008; LOISEL et al., 1994; SCHULTZ et al., 2005). Em menos de dois anos tem sido construída uma rede envolvendo pessoas e instituições, acelerando o processo de construção/formação do Programa de Retorno ao Trabalho de Trabalhadores com LER/DORT do Cesat/Bahia.

Programa de Retorno ao Trabalho de Trabalhadores

Há um consenso na literatura especializada quanto à necessidade de compor Programas de Retorno ao Trabalho (PRT) para patologias musculoesqueléticas abrangentes, integrando as dimensões biopsicossociais do adoecimento, envolvendo os diferentes atores sociais interessados e respeitando as dinâmicas presentes nas relações institucionais e interinstitucionais (SCHULTZ et al., 2005; YOUNG et al., 2005).

A própria definição de "retorno ao trabalho", como um resultado final a ser obtido ou como um processo dinâmico que tem como ponto de partida o momento muito anterior ao retorno ao trabalho e se estende por toda a trajetória do trabalhador envolvido, constitui debate recentemente valorizado, justificado pela necessidade de incluir, no conjunto de recursos mobilizados, indicadores de avaliação intermediários, sensíveis às inúmeras

\footnotetext{
${ }^{5}$ O grupo de pesquisa Saúde, Trabalho e Funcionalidade está cadastrado no CNPQ desde 2006 e vem trabalhando na elaboração de um Protocolo de Avaliação da Funcionalidade de Trabalhadores com LER/DORT. Trata-se de um grupo de docentes e pesquisadores do Departamento de Medicina Preventiva e Social da Faculdade de Medicina da Bahia/UFBA e dos Cursos de Terapia Ocupacional e Psicologia da Escola Bahiana de Medicina e Saúde Pública, que tem estudado a CIF e as suas aplicações para o campo da Saúde dos Trabalhadores e promovido atividades de capacitação de recursos humanos voltadas para a reabilitação de trabalhadores. Em 2007, elaborou o Core Set LER/DORT (LIMA et al., 2008) e organizou o Protocolo de Avaliação da Funcionalidade de Trabalhadores com LER/DORT em fase de validação.
} 
variações que podem ocorrer nas diferentes etapas e que podem constituir barreiras para o sucesso efetivo deste processo. Nesta direção, aponta-se, também, a necessidade de reconhecer e definir adequadamente as etapas/fases dos PRTs, as ações a serem efetivadas e os resultados esperados em cada uma delas (YOUNG et al., 2005).

Os modelos emergentes de retorno ao trabalho, como no caso do Modelo Ecológico, o de Gerenciamento de Caso e o Modelo Econômico, valorizam abordagens multiprofissionais e interdisciplinares, com foco na interação entre o trabalhador doente e os múltiplos sistemas no interior dos quais os atores sociais interessados mobilizam-se de forma dinâmica, perpassados por contingências descritas como relacionadas a fatores pessoais relativos ao trabalhador doente (microssistemas), fatores relacionados aos ambientes de trabalho, assistência à saúde ou seguro de saúde (mesossistemas) e fatores relacionados à dimensão econômica, social ou legislativa (macrossistemas) (SCHULTZ et al., 2005).

Para Loisel et al. (2001a; 2005), as ações e atitudes dos atores sociais chaves no local de trabalho/empresa, no sistema de saúde e no sistema de compensação são definidoras para o processo de incapacidade ocupacional. Integram o modelo proposto por este autor, os diferentes sistemas acima mencionados e os níveis hierarquizados que tentam integrar desde as demandas afetivas, cognitivas e físicas, os vários níveis organizacionais do sistema de saúde, do local de trabalho e do sistema de compensação, com base no contexto sociocultural.
O PRT aqui proposto incorpora as principais orientações dos modelos internacionais emergentes, tendo como referência o paradigma da Vigilância à Saúde que busca articular o "conjunto de ações de saúde (prevenção, proteção, recuperação e reabilitação) sob indivíduos e sobre o ambiente visando o controle de agravos (...) e a proteção de grupos vulneráveis” (PAIM, 1999 p. 481) dispersos em diferentes instituições e setores da sociedade, implicando em ações setoriais, intersetoriais e "extra-setoriais para enfrentar problemas contínuos em um determinado território” (Idem Ibidem, 1999).

Este projeto em construção incorpora diferentes tecnologias materiais e não materiais mobilizadas com base na experiência acumulada no campo da saúde do trabalhador do SUS e utilizadas nos sistemas mencionados (micro, meso e macrossistemas), respeitando especificidades do contexto socioeconômico brasileiro, que agrega dificuldades para a implantação de ações de saúde com este grau de complexidade.

A seguir, a Figura 1 apresenta um esquema de interação dos atores envolvidos no Programa de Reabilitação de Trabalhadores com LER/DORT do Cesat. Nesta representação busca-se chamar a atenção do potencial mobilizador-integrador da problemática do trabalhador com LER/DORT a partir de uma abordagem voltada para a construção/implantação de processos de interlocução para a produção de tecnologia e de conhecimento e do promotor de mudanças organizacionais. São valorizadas as dinâmicas internas e externas aos atores sociais, mediadas por espaços de negociação e pela definição de prioridades em contínuo processo de avaliação.

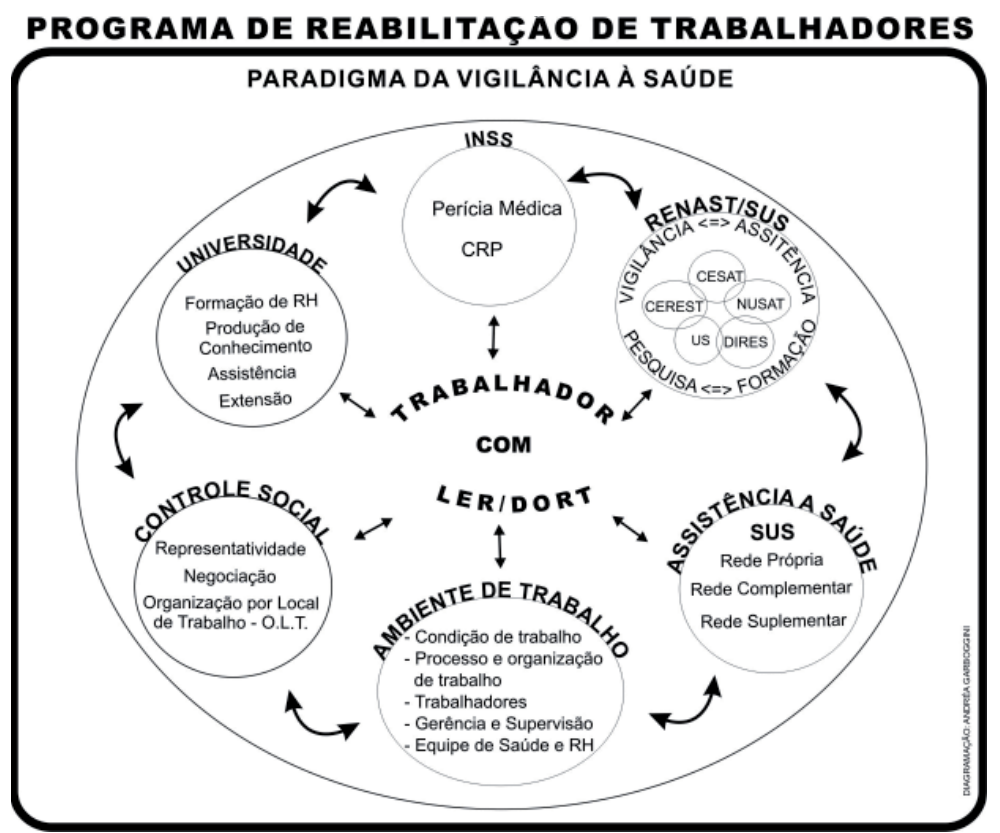

Figura 1 Esquema de interação dos atores envolvidos no Programa de Reabilitação de Trabalhadores proposto pelo Cesat Siglas: INSS - Instituto Nacional de Seguridade Social; Renast - Rede Nacional de Atenção Integral à Saúde do Trabalhador; CRP - Centro de Reabilitação Profissional; Cesat - Centro Estadual de Referência em Saúde do Trabalhador; Cerest - Centro Regional em Saúde do Trabalhador; Nusat - Núcleo de Saúde do Trabalhador; Dires - Diretoria Regional de Saúde; US - Unidade Sentinela; SUS - Sistema Único de Saúde; RH - Recursos Humanos 
Este percurso está sendo conduzido com base em uma modalidade de investigação participante (MINAYO, 2006) com o objetivo de implantar não só um programa pré-formatado, mas de construí-lo, coletivamente, ao tempo em que são formados recursos humanos e espaços institucionais incluindo um programa de reabilitação de trabalhadores, que prevê novas práticas, novos praticantes e novas relações interinstitucionais.

O projeto piloto do PRT foi concebido em três fases (Quadro 1) que preveem desde negociações preliminares com os atores sociais, formação de recursos humanos, até intervenção e acompanhamento do processo de retorno ao trabalho de trabalhadores com LER/DORT (YOUNG et al. 2005). Este tipo de intervenção tem favorecido a interlocução de um grupo de atores com distintos interesses no processo de tratamento-reabilitação de trabalhadores, permitindo a criação de espaços de negociação para a solução contínua de problemas, respeitando a autonomia de cada ator social (trabalhadores, empregadores, Previdência Social, Cesat/Renast/SUS etc.).

Para o registro e a documentação deste processo, estão sendo utilizados múltiplos recursos metodológicos: observação participante com registro em diário de campo; entrevistas em profundidade; registros fotográficos e filmagem dos processos produtivos e de atividades realizadas com os trabalhadores; grupo focal; registro das reuniões da equipe técnica condutora do projeto para discussão e busca de soluções de problemas; e outros para apreender o processo de construção desta intervenção.

A seguir, serão apresentados os passos adotados para a execução deste projeto piloto. A primeira fase, chamada de negociação preliminar, junto aos atores sociais envolvidos no processo de reabilitação de trabalhadores, foi iniciada em 2008. Nesta fase, foram definidos os critérios de elegibilidade da empresa para participação do projeto, incluindo número de trabalhadores atingidos ou expostos, importância econômica da atividade produtiva, existência de demanda organizada, factibilidade e possibilidade de êxito na adoção de medidas de prevenção (BAHIA, 2002).

A empresa selecionada para o projeto piloto é uma distribuidora de alimentos, de economia mista, que possui 282 lojas no estado da Bahia, além de centrais de distribuição, frigoríficos, prédios administrativos, totalizando 3.199 funcionários, dos quais 415 (13\%) encontravam-se afastados do trabalho no momento do início deste projeto. Destes, 151 (4,72\%) com algum diagnóstico de desordens musculoesqueléticas. Vários destes trabalhadores afastados (31 trabalhadores) foram acompanhados pelo Cesat ao longo dos últimos quatro anos (2005 a 2008). Ainda nesta fase, foi realizado o diagnóstico preliminar com o estudo das características dos trabalhadores afastados e a seleção dos trabalhadores que participariam do PRT/Cesat.

O diagnóstico preliminar foi elaborado com base na avaliação dos prontuários dos 111 trabalhadores afastados pela Previdência Social com diagnóstico compatível com LER/DORT. Dentre os trabalhadores afastados, apenas um tinha menos que 30 anos, 64 estavam entre 30 e 49 anos e 32 , acima de 50 anos. A maioria dos trabalhadores afastados realizava funções relacionadas à atividade de operador de caixa: 64 trabalhadores como auxiliar de operação, 20 como fiscal de caixa, 8 como operadores de caixa e 4 como caixas, totalizando 96 trabalhadores do total de 111 afastados. Quanto ao tempo de afastamento, chama atenção que apenas 14 apresentam menos de 1 ano de afastamento, 40 de 1 a 3 anos, 34 entre 3 e 6 anos e 17 com tempo superior a 6 anos. Com base nestes dados, elegeram-se como critérios de inclusão destes trabalhadores no PRT: o desejo de participar do programa, estar lotado em uma das lojas de Salvador, ter a função relacionada à atividade de caixa e ter o diagnóstico de LER/DORT. Foram selecionados 40 trabalhadores.

Também foi realizado o levantamento de trabalhadores que estavam em atividade na empresa, mas que tinham apresentado atestado médico com até 15 dias de afastamento do trabalho no ano de 2009. Assim, foram relacionados 97 trabalhadores com atestados de curta duração e aplicados os critérios de inclusão no PRT. Destes, 17 foram selecionados.

Como um dos critérios de inclusão é o desejo em participar do programa, o total de trabalhadores selecionados foi de 57 , na perspectiva de alcançar um grupo final de 30 participantes. Os selecionados serão avaliados utilizando-se o Protocolo de Avaliação de Funcionalidade (descrito abaixo), com base no qual será elaborado o plano de reabilitação. Este subsidiará ações de articulação com serviços de saúde da rede SUS para o suporte terapêutico necessário a cada trabalhador.

Nesta fase, têm sido feitos vários contatos com a empresa e com o INSS com o objetivo de apresentar a proposta deste projeto, formalizar parcerias, compor equipe condutora com membros da empresa, representantes dos trabalhadores, INSS (perícia médica e Centro de Reabilitação Profissional - CRP) e equipe técnica do Cesat.

No contato mantido com o INSS, foi encaminhada uma proposta para o estabelecimento de convênio de cooperação técnica com o Cesat para formalização da parceria e composição da equipe condutora por técnicos do CRP e da perícia médica.

Para a finalização desta primeira fase, foi realizado um momento de sensibilização envolvendo a alta gestão da empresa, os trabalhadores do nível de gerência e os supervisores, além da equipe condutora do projeto, tendo sido apresentado todo o andamento previsto para este projeto em suas diversas etapas, fomentando o envolvimento de todos, ressaltando os pressupostos teórico-práticos norteadores deste programa. Como fruto deste momento, foi definida a elaboração de um termo de compromisso a ser firmado entre o Cesat, a empresa e a representação sindical, estabelecendo, dentre outras coisas, garantias de estabilidade no emprego, durante o desenvolvimento de todas as etapas, para os trabalhadores que aderirem ao projeto. 
Quadro 1 Fases do Programa de Retorno ao Trabalho - PRT: Projeto Piloto

\begin{tabular}{|c|c|c|}
\hline Fases & Ações & Detalhamento \\
\hline \multirow{3}{*}{$1^{\underline{a}}$ - Inicial } & Negociação inicial & $\begin{array}{l}\text { - Definição de critérios de elegibilidade da empresa para o programa; } \\
\text { - Apresentação de proposta à empresa; } \\
\text { - Formalização - termo de convênio; } \\
\text { - Envolvimento dos representantes dos trabalhadores; } \\
\text { - Composição da equipe condutora: membros da empresa, representan- } \\
\text { tes dos trabalhadores, equipe técnica do PRT/Cesat. }\end{array}$ \\
\hline & $\begin{array}{l}\text { Diagnóstico da situação } \\
\text { de saúde }\end{array}$ & $\begin{array}{l}\text { - Estudo das características dos trabalhadores afastados: tempo de afas- } \\
\text { tamento, escolaridade, tempo de serviço, idade, atestados médicos de } \\
\text { curta duração; } \\
\text { - Eleição dos trabalhadores para o PRT. }\end{array}$ \\
\hline & Sensibilização & $\begin{array}{l}\text { - Sensibilização e envolvimento da alta gestão, gerência e supervisores } \\
\text { da empresa. }\end{array}$ \\
\hline \multirow{4}{*}{$\begin{array}{l}2^{2}-\text { - Treinamento } \\
\text { Avaliação }\end{array}$} & Capacitação geral & $\begin{array}{l}\text { - Treinamento sobre reabilitação de trabalhadores: processo de trabalho, } \\
\text { programas de retorno ao trabalho, ergonomia, avaliação de funcionalidade; } \\
\text { - Tutorial, atividades presenciais e em campo, aplicação de método. }\end{array}$ \\
\hline & $\begin{array}{l}\text { Avaliação da funcionali- } \\
\text { dade dos trabalhadores } \\
\text { afastados }\end{array}$ & $\begin{array}{l}\text { - Aplicação do protocolo de funcionalidade, avaliação de habilidades, } \\
\text { levantamento de perspectivas e demandas, formulação de plano de } \\
\text { reabilitação, identificação de opções de trabalho factíveis, garantia de } \\
\text { suporte junto à equipe de trabalho. }\end{array}$ \\
\hline & $\begin{array}{l}\text { Avaliação da situação de } \\
\text { trabalho }\end{array}$ & $\begin{array}{l}\text { - Visitas ao local de trabalho; } \\
\text { - Análise ergonômica do trabalho; } \\
\text { - Elaboração de proposta de intervenção. }\end{array}$ \\
\hline & $\begin{array}{l}\text { Apresentação da pro- } \\
\text { posta à alta gestão da } \\
\text { empresa }\end{array}$ & $\begin{array}{l}\text { - Negociação do retorno ao trabalho dos trabalhadores no PRT; } \\
\text { - Negociação sobre as intervenções necessárias no processo de trabalho. }\end{array}$ \\
\hline \multirow{4}{*}{$3^{\underline{a}}$ - Intervenção } & $\begin{array}{l}\text { Acompanhamento do } \\
\text { retorno ao trabalho }\end{array}$ & $\begin{array}{l}\text { - Avaliação da adequação da capacidade trabalho-desempenho; } \\
\text { - Identificação dos problemas e intervenção: modificação das tarefas, } \\
\text { do plano de trabalho, da tecnologia assistiva, estímulo de habilidades, } \\
\text { redefine metas, definição da progressão de metas de produção; } \\
\text { - Suporte terapêutico. }\end{array}$ \\
\hline & $\begin{array}{l}\text { Implantação das inter- } \\
\text { venções no posto de } \\
\text { trabalho/situação de } \\
\text { trabalho }\end{array}$ & $\begin{array}{l}\text { - Explicação sobre as principais tarefas e/ou etapas do processo de } \\
\text { trabalho que deverão ser modificadas; } \\
\text { - Proposição de soluções negociadas; } \\
\text { - Avaliação de viabilidade das soluções e estabelecimento de prioridades } \\
\text { baseados na severidade e no risco à saúde, impacto sobre o processo } \\
\text { produtivo como um todo. }\end{array}$ \\
\hline & Manutenção & $\begin{array}{l}\text { - Acompanhamento do desempenho do trabalhador com identificação } \\
\text { de ganho de autonomia, estabilização ou regressão; } \\
\text { - Reconhecimento do grau de satisfação do trabalhador com o PRT; } \\
\text { - Identificação de novos objetivos e perspectivas profissionais; } \\
\text { - Acompanhamento das demandas de saúde. }\end{array}$ \\
\hline & Avançado & $\begin{array}{l}\text { - Identificação de novas habilidades/vocações; } \\
\text { - Formulação de plano para alcançar novas colocações; } \\
\text { - Identificação de oportunidades compatíveis; } \\
\text { - Acompanhamento de novo processo de mudança. }\end{array}$ \\
\hline
\end{tabular}


Para a segunda fase do projeto piloto estão previstas capacitações e treinamentos de toda a equipe condutora, tanto na temática geral de reabilitação de trabalhadores em programas de retorno ao trabalho, como na aplicação do Protocolo de Avaliação de Funcionalidade e na abordagem ergonômica voltada para reabilitação.

Os trabalhadores selecionados serão submetidos à avaliação de funcionalidade com o objetivo de levantamento de perspectivas e demandas, formulação de plano de reabilitação, identificação de opções de trabalho factíveis, avaliação de habilidades, garantia de suporte junto à equipe de trabalho. Posteriormente, será realizada avaliação individual das situações de trabalho, com visitas ao local do mesmo, à análise ergonômica e à elaboração de proposta de intervenção na organização deste.

Este processo de treinamento será conduzido com base em referência teórica que reconhece a aprendizagem como processual e assume a problematização como uma referência na construção de novos saberes e práticas. A problematização tem sua origem nos estudos de Paulo Freire, enfatizando que os problemas a serem estudados precisam valer-se de um cenário real (CYRINO; TORALLES-PEREIRA, 2004).

A conclusão da segunda fase será feita com a apresentação da proposta de retorno ao trabalho dos trabalhadores avaliados à alta gestão da empresa, implicando negociações necessárias a este retorno.

A terceira fase deste projeto, chamada de Fase de Intervenção e Acompanhamento, prevê o acompanhamento do retorno ao trabalho de trabalhadores, individualmente e em grupo. Serão observados os seguintes aspectos: avaliação da adequação da capacidade trabalho-desempenho; identificação dos problemas e intervenção no processo de trabalho (biomecânica e organização do trabalho: modificação das tarefas, do plano de trabalho, introdução de tecnologia assistiva, estimulação de habilidades, redefinição de metas, definição de progressão de metas de produção). Será também mantido suporte terapêutico voltado para o manejo de recursos de controle da dor, identificação de reagudizações ou de novas demandas físicas e psíquicas.

A implantação das intervenções ergonômicas propostas pela equipe multiprofissional, após a avaliação das situações de trabalho, passará por apresentação e explicação das principais tarefas e/ou etapas do processo que deverá ser modificado; proposição de soluções negociadas; avaliação de viabilidade das soluções, do estabelecimento de prioridades - baseadas na severidade e no risco à saúde - e do impacto sobre o processo produtivo como um todo. Este processo será acompanhado por todos os atores sociais envolvidos sob a coordenação do Cesat/Bahia.

A manutenção desta intervenção será feita a partir do acompanhamento do desempenho dos trabalhadores, tendo como principal referência o ganho progressivo de autonomia, a estabilização do quadro clínico e o envolvimento motivacional, com reconhecimento do grau de satisfação do trabalhador com o PRT, identificação de novos objetivos e perspectivas profissionais. Este processo também prevê o acompanhamento de demandas de saúde relacionadas ou não às LER/DORT.

As ações avançadas do PRT incluem identificação de novas habilidades/vocações, formulação de plano compartilhado com a equipe técnica para alcançar novas colocações e desenhar novos projetos, identificar oportunidades compatíveis e sustentar o acompanhamento de novo processo de mudança.

\section{A CIF e o protocolo de avaliação da funcionalidade de trabalhadores com LER/DORT}

A CIF foi criada pela Organização Mundial de Saúde, no ano de 2001, com objetivo de "proporcionar uma linguagem unificada e padronizada e uma estrutura que descreva a saúde e os estados relacionados à saúde" (ORGANIZAÇÃO MUNDIAL DA SAÚDE, 2003, p. 13). Tem sido utilizada na discussão da funcionalidade do ponto de vista clínico ou como modelo teórico em estudos sobre dor crônica e desordens musculoesqueléticas (CHEN et al., 2003), mas também tem sido questionada sua aplicabilidade em programas de retorno ao trabalho (YOUNG et al., 2005).

A CIF propõe um modelo de funcionalidade e incapacidade que tem em seu centro (Figura 2) a atividade humana, aqui entendida como o resultado da interação de fatores ligados à condição de saúde (funções e estruturas do corpo, atividade/participação) e aos fatores contextuais (ambientais e pessoais). Esta centralidade requer compreender a demanda do sujeito que sofre e o seu entorno (ORGANIZAÇÃO MUNDIAL DA SAÚDE, 2003) e possibilita a ampliação dos recursos terapêuticos, incluindo abordagens não só, nem prioritariamente, sobre o indivíduo ou a doença, mas sobre os obstáculos e os facilitadores ambientais em interação com as restrições da participação social.

O ponto de vista assumido nesta leitura do modelo teórico da CIF reconhece a importância de aprofundar o estudo do cotidiano, incluindo aí o mundo do trabalho, com base em uma abordagem compreensiva orientada pelas ciências sociais que permita uma melhor articulação dos elementos internos e externos a este modelo, presentes na dinâmica social mobilizada em torno do processo de tratamento-reabilitação de trabalhadores.

O Protocolo de Avaliação de Funcionalidade de Trabalhadores com LER/DORT parte do Modelo Teórico da CIF, incorporando outros recursos com base em uma triangulação metodológica que utiliza, de forma combinada, entrevista narrativa, instrumentos validados de avaliação de aspectos pessoais, o Core Set LER/DORT propriamente dito, mapa cognitivo, síntese da funcionalidade e o plano de reabilitação (LIMA et al., 2008).

A triangulação metodológica tem sido proposta para favorecer o diálogo entre áreas de conhecimento, sendo capaz de viabilizar o entrelaçamento entre teoria e prática e de agregar múltiplos pontos de vista - seja das variadas formulações teóricas utilizadas pelos pesquisadores, ou da visão de mundo dos informantes da pesquisa - utilizados de modo articulado no estudo, bem como na perspectiva de combinar múltiplas estratégias de pesquisa capazes de apreender melhor objetos complexos (MINAYO et al., 2005). 


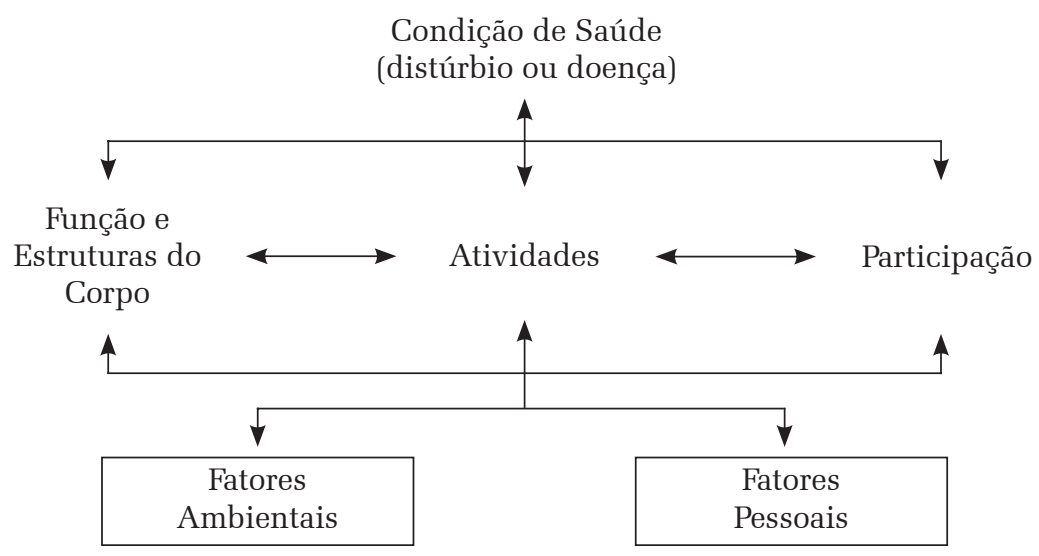

Figura 2 Modelo de entendimento da funcionalidade de acordo com a CIF

Fonte: CIF (ORGANIZAÇÃO MUNDIAL DA SAÚDE, 2003).

Quadro 2 Etapas do protocolo de avaliação de funcionalidade de trabalhadores com LER/DORT

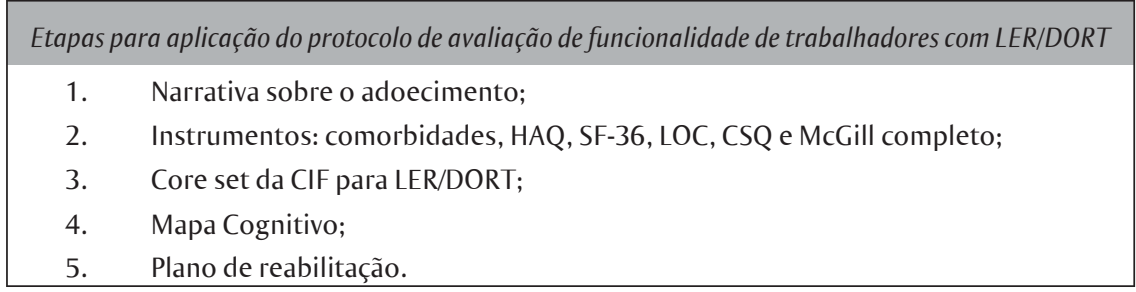

Fonte: LIMA et al., 2008

A narrativa neste protocolo cumpre o importante papel de fornecer elementos para o processo de reabilitação, que não seriam possíveis de serem identificados através de instrumentos estruturados ou semiestruturados. São experiências compartilhadas e construídas dialogicamente, contadas por conversas interconectadas de várias pessoas, histórias cujos referenciais são as experiências das pessoas e não dos narradores. Elas falam, dentre outros elementos, de relações sociais conflitantes, de relações de poder e apresentam configurações que dão ordenamento coerente à vida cotidiana. Desta forma, o que se pretende é que as narrativas associem-se a outros elementos avaliativos para subsidiar uma maior compreensão sobre a reabilitação de trabalhadores com LER/DORT com base na valorização da voz do trabalhador adoecido em sua busca por cuidado (GOOD, 1994).

Vale ressaltar que a síntese da funcionalidade e plano de reabilitação é a sistematização dos achados, a organização das informações e a apresentação das propostas de ação. Neste momento, é retomada a narrativa do adoecimento enquanto eixo interpretativo, sobre o qual são acrescidos elementos identificados com os demais instrumentos. O plano de reabilitação tem como elementos centrais as demandas identificadas e valorizadas pelo trabalhador, as perspectivas apontadas para a adesão ao programa de retorno ao trabalho, o resultado da avaliação do processo de trabalho e a avaliação clínica.

A utilização deste protocolo, no contexto de um programa de retorno ao trabalho, tem possibilitado aos profissionais envolvidos e ao trabalhador acolhido conhecer e reconhecer não só as limitações, mas, principalmente, as possibilidades físicas, psíquicas e sociais do trabalhador. Ou seja, inclui a identificação dos obstáculos e dos facilitadores para o processo de reabilitação, a serem discutidos tanto com o trabalhador, como com os demais atores sociais envolvidos, e permite a ampliação da participação destes no processo de tratamento-reabilitação.

As experiências de aplicação do protocolo, realizadas no Cesat/BA nos últimos dois anos, têm fomentado discussões em todas as etapas do processo, intensificando o diálogo entre centro de referência, instituições de ensino superior, serviços de saúde, empresa e previdência social. Têm funcionado como um motivador de reflexão sobre a complexidade das LER/DORT, com base em uma escuta culturalmente sensível sobre os limites e as possibilidades das práticas de saúde dos diversos atores sociais envolvidos no processo de tratamento-reabilitação de trabalhadores. 


\section{Considerações finais}

O processo de concepção e implantação do programa de reabilitação de trabalhadores com LER/DORT do Cesat/BAHIA tem proporcionado tanto o amadurecimento teórico do grupo técnico que o tem desenhado, como também o fortalecimento das interações institucionais necessárias para o desenvolvimento de um projeto deste porte. Ainda é um processo inicial que conta com a motivação do grupo e com as conjunturas política e institucional favoráveis. O grupo reconhece a necessidade de se conduzir uma dupla avaliação voltada para

\section{Referências}

BAHIA. Secretaria de Saúde. Superintendência de Vigilância e Proteção da Saúde. Centro de Estudo da Saúde do Trabalhador. Manual de normas e procedimentos técnicos para a vigilância da saúde do trabalhador. 3. ed. Salvador: Cesat/SESAB, 2002.

CHEN, J. et al. Prevalence of lower extremity pain and its association with functionality and quality of life in elderly women in Australia. The Journal of Rheumatology, Canada, v. 30, n. 12, p. 2689-2693, Dec. 2003.

CYRINO, E. G.; TORALLES-PEREIRA, M. L.

Trabalhando com estratégias de ensino-aprendizado por descoberta na área de saúde: a problematização e a aprendizagem baseada em problemas. Cadernos de Saúde Pública, Rio de Janeiro, v. 20, n. 3, p. 780-788, maio/jun. 2004.

DURAND, M. J. et al. Conctructing the program impact theory for evidence-based work rehabilitation program for workers with low back pain. Work, Netherlands, v. 21, n. 3, p. 233-242, 2003.

GOOD, B. J. The body, illness experience, and the lifeworld: a phenomenological account of chronic pain. In: Medicine, rationality, and experience: an anthropological perspective. Cambridge: Cambridge University Press, 1994. p. 116-134.

LIMA, M. A. et al. Avaliação da funcionalidade dos trabalhadores com LER/DORT: a construção do Core Set da CIF para LER/DORT. Acta Fisiatrica, São Paulo, v. 15, n. 4, p. 229-235, dez. 2008.

LOISEL, P. et al. Management of occupational back pain: the sherbrooke model. Results of a pilot and feasibility study. Occupational and Environmental Medicine, England, v. 51, n. 9, p. 597-602, Sep. 1994.

. et al. Implementation of a participatory ergonomics program in the rehabilitation of workers suffering from subacute back pain. Applied Ergonomics, England, v. 32 n. 1, p. 53-60, Fev. 2001b.

. et al. Disability prevention. The new paradigm of management of occupational back pain. avaliar a tecnologia produzida nas fases de implantação e para avaliar as estratégias utilizadas para promover a interação entre os atores sociais interessados no retorno ao trabalho a partir de diferentes pontos de vista. Apesar de o processo estar na fase inicial, esta intervenção, que prioriza o estabelecimento de inter-relações institucionais, tem proporcionado reflexões sobre as ações até então desenvolvidas pelos diversos atores envolvidos no processo de reabilitação, que podem contribuir para a discussão sobre a reabilitação de trabalhadores na busca de um modelo factível a ser implantado na Renast/SUS/BAHIA com maior resolutividade que os modelos tradicionais de reabilitação de trabalhadores.
Disease Management and Clinical Outcomes, United States, v. 9, n. 7, p. 351-360, 2001a.

. et al. Prevention of work disability due to musculoskeletal disorders: the challenge of implementing evidence. Journal of Occupational Rehabilitation, Netherlands, v. 15, n. 4, p. 507-524, Dec. 2005.

MAENO, M. Reinserção de trabalhadores com lesões por esforços repetitivos no mercado de trabalho. 2001. 117 f. Dissertação. (Mestrado)-Faculdade de Saúde Pública. Universidade de São Paulo, São Paulo, 2001.

MAENO, M.; TAKAHASHI, M. A. C.; LIMA, M. A. G. Reabilitação profissional como política de inclusão social. Acta Fisiátrica, São Paulo, v. 16, n. 2, p. 53-58, jun. 2009.

MINAYO, M. C. S. O desafio do conhecimento: pesquisa qualitativa em saúde. 9. ed. São Paulo: Hucitec, 2006.

MINAYO, M. C; ASSIS, S. G; SOUZA, E. R. Avaliação por triangulação de métodos: abordagem de programas sociais. Rio de Janeiro: Fiocruz, 2005.

ORGANIZAÇÃO MUNDIAL DE SAÚDE. CIF: classificação internacional de funcionalidade, incapacidade e saúde. São Paulo: Edusp, 2003.

PAIM, J. S. A reforma sanitária e os modelos assistenciais. In: ROUQUAYROL, M. Z.; ALMEIDA FILHO, N. (Org.). Epidemiologia e saúde. 5.ed. Rio de Janeiro: MEDSI, 1999.

. Modelos assistenciais: reformulando o pensamento e incorporando a proteção e a promoção de saúde. In: . Saúde política e reforma sanitária. Salvador: CEPSISC, 2002.

ROSSI, D. et al. Habilitação e reabilitação profissional: abordagem interdisciplinar, intersetorial e interinstitucional. Documento para discussão pública. Seminário reabilitação profissional pública, um direito do cidadão. São Paulo: 2007. Disponível em: $<$ www.spbancarios.com.br/download/17/proposta_rp_ out2007.pdf > . Acesso em: 23 jun. 2008. 
SCHULTZ, I. Z. et al. Models of return to work for musculoskeletal disorders. Journal of Occupational Rehabilitation, Netherlands, v. 17, n. 2, p. 327-352, 2005.

SOUZA, N. S. S. ESTRELA, T. Evolução da morbidade e do perfil dos trabalhadores atendidos em um centro de referência de saúde do trabalhador no estado da Bahia no período de 1991 a 2000. In: Cadernos de Saúde do Trabalhador, Bahia, Salvador: Cesat, 2003, p. 23-31.

TAKAHASHI, M. A. B. C.; CANESQUI, A. M. Pesquisa avaliativa em reabilitação profissional: a efetividade de um serviço em desconstrução. Cadernos de Saúde Pública, Rio de Janeiro, v. 19, n. 5, p. 1473-1483, set./ out. 2003.
TAKAHASHI, M. A. B. C.; IGUTI, A. M. As mudanças nas práticas de reabilitação profissional da Previdência Social no Brasil: modernização ou enfraquecimento da proteção social? Cadernos de Saúde Pública, Rio de Janeiro, v. 24, n. 11, p. 2661-2670, Nov. 2008.

YOUNG, A. E. et al. A developmental conceptualization of return to work. Journal of Occupational Rehabilitation, Netherlands, v. 15, n. 4, p. 557-568, Dec. 2005.

XAVIER, C. GUIMARÃES, C. PINHEIRO, R. Uma semiótica da integralidade: o signo da integralidade e o papel da comunicação. In: MATTOS, R. A. (Org.). Cuidado: as fronteiras da integralidade. Rio de Janeiro: HUCITEC, 2004. p. 133-155. 